\title{
Evaluación preanestésica del paciente gran quemado agudo adulto
}

\author{
CLARA LUXORO V. ${ }^{1}$
}

1 Hospital de Urgencia Asistencia Pública, Profesor adjunto, Universidad de Santiago.

\section{Introducción}

Las quemaduras son lesiones producidas en los tejidos vivos debido a la acción de diversos agentes físicos, químicos y biológicos, que provocan alteraciones que van desde un simple eritema hasta la destrucción total de las estructuras $^{1}$.

El paciente gran quemado es aquel que se incluye en cualquiera de las siguientes categorías ${ }^{1,2,8}$ :

- Aquellos con un índice de gravedad $>70$ puntos o con quemaduras $\mathrm{AB}$ o $\mathrm{B}>20 \%$ de superficie corporal.

- Aquellos > 65 años con $10 \%$ o más de quemadura $\mathrm{AB}$ o $\mathrm{B}$.

- Aquellos con quemaduras respiratorias por inhalación de humo.

- Aquellos con quemaduras eléctricas por alta tensión.

- Quemados politraumatizados.

- Quemados con patologías graves asociadas.

La mejoría persistente de la sobrevida que han experimentado los grandes quemados a partir de la segunda mitad del siglo XX se atribuye a diversos factores $2,4,6,13$ :

- Posibilidad de reanimación con fluidos.

- Avances en el soporte nutricional.

- Uso de antibióticos más efectivos.

- Avances en el tratamiento de la injuria inhalatoria.

- Desarrollo de centros para el cuidado de quemados.
- Resección y cobertura precoz de las quemaduras.

En el Servicio de Quemados del Hospital de Urgencias Asistencia Pública (HUAP), durante el año 2011, los pacientes gran quemados, todos adultos mayores de 15 años, se sometieron en promedio a 4,2 cirugías durante su fase aguda (desde el accidente hasta la resección, injerto y curación de las quemaduras), excluyendo las requeridas para reparación de secuelas.

La evaluación preanestésica corresponde al proceso de entrevista y estudio clínico que precede a la administración de anestesia que permitirá la realización de una intervención quirúrgica, procedimiento diagnóstico o terapéutico ${ }^{3}$.

Se trata de un proceso complejo, multidisciplinario, donde en ocasiones pueden interactuar diferentes especialistas, como médicos generales, anestesiólogos, cirujanos, internistas de diferentes subespecialidades, intensivistas, enfermeras y diversos servicios de apoyo (laboratorio, banco de sangre, imagenología). Todos ellos deben contribuir a asegurar el mejor resultado posible, aplicando cuidados de calidad, a costos razonables ${ }^{3}$.

Durante este proceso, debe tenerse en consideración información proveniente de múltiples fuentes:

- Ficha Clínica: historia clínica, signos vitales, indicaciones médicas.

- Entrevista al paciente y/o a sus familiares.

- Examen físico.

- Exámenes de laboratorio.

Correspondencia a:

Dra. Clara Luxoro V.

cluxoro@vtr.net 
- Exámenes de imagenología.

- Exámenes especiales.

- Interconsultas a otros especialistas: broncopulmonares, cardiólogos, nefrólogos, hematólogos, endocrinólogos, neurólogos, traumatólogos, ginecólogos, etc., considerando la comorbilidad del paciente y el daño de órganos como consecuencia de su patología aguda.

El objetivo de la evaluación preanestésica es realizar la intervención quirúrgica o el procedimiento diagnóstico o terapéutico indicados, en las mejores condiciones posibles del estado de salud del paciente, considerando en todo momento la premura con que debe realizarse la cirugía, de forma de obtener el mayor beneficio posible en relación a su condición de salud ${ }^{3}$.

Existen procedimientos quirúrgicos cuya premura nos limitará bastante el tiempo de estudio y preparación del paciente para la cirugía: aseo quirúrgico inicial, escarotomía, fasciotomía, descompresión de tronco. Aún así, se recomienda practicarlos una vez estabilizado el paciente desde el punto de vista hemodinámico (etapa de shock superada, reposición efectiva de fluidos), con evacuación vesical funcionante, y dolor abolido ${ }^{1}$.

Otras cirugías son planificadas (escarectomías tangenciales o a fascia con coberturas transitorias o permanentes, aseos quirúrgicos, amputaciones) y permiten un estudio y preparación más profun$\operatorname{dos}^{1}$.

El tratamiento quirúrgico precoz de las quemaduras con escarectomía y cobertura cutánea, determinará menor dolor, más rápida recuperación, disminución de requerimientos energéticos basales, disminución de infecciones de quema- duras, disminución de secuelas y mejoría de la sobrevida ${ }^{2,4,5}$. Por esta razón se espera que todo el equipo de profesionales que atiende al paciente gran quemado, entre ellos los anestesiólogos, seamos facilitadores de la aplicación de este concepto. Es importante enfatizar una resección precoz del tejido quemado no viable, pues este puede contener gérmenes patógenos y/o desencadenar la liberación de mediadores inflamatorios que afectan el metabolismo y la función cardiopulmonar ${ }^{6}$.

La piel es el mayor órgano del cuerpo humano. En adultos, su peso aproximado corresponde a $4,2 \mathrm{~kg}$ y da cuenta de un 14 a $17 \%$ del peso corporal. Su volumen es $3,6 \mathrm{~L}$, su área $1,8 \mathrm{~m}^{2}$ Tiene un espesor variable de 0,5 a $4 \mathrm{~mm}$. Su destrucción compromete todas sus funciones (Tabla $1)$.

La piel protege, intercambia sustancias, regula, metaboliza, informa, identifica, interactúa socialmente, discrimina.

\section{Tareas incluidas durante la evaluación preanestésica del paciente gran quemado}

\section{Identificación y datos generales y antropométricos del paciente \\ Nombre completo \\ Sexo \\ Edad \\ Peso (antes y después del accidente) \\ Talla \\ IMC \\ Hábitos (tabaquismo, alcohol, drogas) \\ Alergias}

Tabla 1. Funciones de la piel*

1. Protección frente a elementos ambientales (radiaciones, irritación mecánica o trauma)
2. Inmunológica: barrera frente a organismos patógenos (sudor y sebo son bacteriostáticos)
3. Homeostasis de fluidos y electrolitos: ayuda a mantener concentraciones de proteínas y electrolitos, limitando la evaporación
4. Termoregulación: ayuda al control de pérdidas de calor a través de la sudoración y de la regulación vasomotora del flujo
sanguíneo superficial
5. Sensibilidad: una variada y extensa clase de receptores (calor, frío, dolor, tacto, presión), aportan información ambiental
6. Metabólica: síntesis de vitamina D y excreción de solutos y líquidos
7. Social: la apariencia de la piel influencia con fuerza la imagen y las interacciones sociales

*Adaptada de Williams WG, Phillips LG. Patofisiología de la quemadura. En Herndon DN, ed. Total Burn Care, Primera edición. Londres: WB Saunders, 1996: 64. 


\section{Determinación de características de las quemaduras del paciente}

Es necesario determinar:

\section{Extensión quemaduras}

El \% total de superficie quemada se utiliza para $^{6}$ :

- Determinar la gravedad y el pronóstico del paciente (Índice de Gravedad, ABSI (Abbreviated Burn Severity Index)).

- Determinar el volumen necesario a aportar durante las primeras $24 \mathrm{~h}$ de producida la quemadura, para tratar el shock inicial. Una fórmula muy utilizada para estimar el volumen a administrar es la fórmula de Parkland:

\section{$4 \mathrm{ml}$ de Ringer lactato $\mathrm{x} \mathrm{kg}$ de peso $\mathrm{x} \%$ total de superficie quemada}

- Determinar el sangrado previsto para una cirugía.

En el paciente adulto se utiliza la regla de los 9 (Tabla 2 y Figura 1) y la regla de la palma de la mano (dedos incluidos), que corresponde a un $1 \%$ de superficie corporal (Figura 2).

Frecuentemente se hace una sobreestimación inicial de la superficie quemada, especialmente al ser efectuada por médicos inexpertos, sobre todo al usar la regla de la palma ${ }^{1,4,6,8}$.

\section{Profundidad de las quemaduras}

Se recomienda el uso de cualquiera de las 3 clasificaciones más conocidas en nuestro país: Be-
Tabla 2. Porcentaje de superficie corporal asignado a áreas anatómicas del paciente adulto, según regla de los 9

\begin{tabular}{|lc|}
\hline Zona Anatómica & $\begin{array}{c}\text { \% Superficie } \\
\text { corporal }\end{array}$ \\
\hline Cabeza & 9 \\
Extremidad superior derecha & 9 \\
Extremidad superior izquierda & 9 \\
Superficie anterior tórax & 9 \\
Superficie anterior abdomen & 9 \\
Superficie posterior tronco & 18 \\
$\begin{array}{l}\text { Superficie anterior extremidad inferior } \\
\text { derecha }\end{array}$ & 9 \\
$\begin{array}{l}\text { Superficie posterior extremidad inferior } \\
\text { derecha }\end{array}$ & 9 \\
$\begin{array}{l}\text { Superficie anterior extremidad inferior } \\
\text { izquierda }\end{array}$ & 9 \\
$\begin{array}{l}\text { Superficie posterior extremidad inferior } \\
\text { izquierda }\end{array}$ & 9 \\
Región perineal & 100 \\
\hline Total & \\
\hline
\end{tabular}

naim, Converse-Smith, o ABA (American Burns Association) (Tabla 3). Debe considerarse que las quemaduras intermedias son evolutivas. La clasificación de Benaim, es la más popular en América Latina. Su fortaleza es poner el énfasis en el pronóstico ${ }^{1}$ (Tabla 4, Figuras 3, 4, 5). El conocimiento de la profundidad de las quemaduras es fundamental tanto para confeccionar el plan quirúrgico como para prepararse para las consecuencias
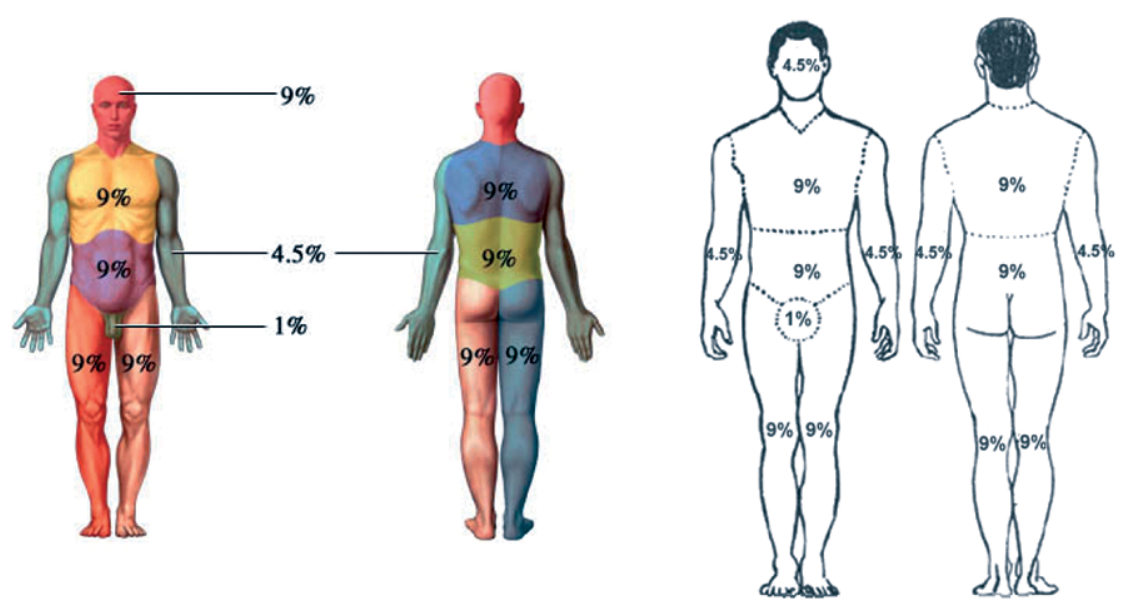

Figura 1. Regla de los 9, tomada del Ministerio de Salud. Guía Clínica Gran Quemado. Minsal, 2007. 


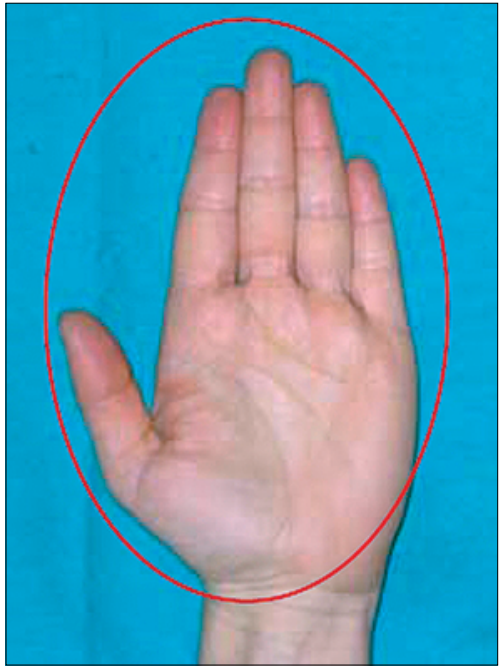

Figura 2. Regla de la palma de las manos, tomada del Ministerio de Salud. Guía Clínica Gran Quemado. Minsal, 2007 (Recordar que para estos efectos la palma de la mano incluye también las superficies palmares de los dedos).

fisiológicas de la agresión quirúrgica. La cirugía de las quemaduras $\mathrm{AB}$ profundas y $\mathrm{B}$, de espesor total, se asocian a sangrados profusos y bruscos ${ }^{6}$.

Algunos autores incluyen en su clasificación de profundidad, las quemaduras de cuarto grado, que corresponderían aquellas que se extienden a músculo y hueso ${ }^{8}$.

\section{Localización de las quemaduras}

Debido al compromiso funcional y estético que podrían determinar, las quemaduras que se ubican en las siguientes zonas tienen una connotación especial ${ }^{1}$ :

- Cara.

- Cuello.

- Manos y pies.

- Pliegues articulares.

- Genitales y periné.

- Mamas.

\section{Edad del paciente}

Las quemaduras de pronóstico más reservado son aquellas que ocurren en pacientes en extremos de la vida ${ }^{1,4}$ :

- $\quad<$ de 2 años.

- $>$ de 60 años.

\section{Sexo del paciente}

El riesgo de morir es aproximadamente el doble en mujeres entre 30 y 59 años comparado con varones de la misma edad ${ }^{4,6}$.

\section{Gravedad del paciente}

Los siguientes factores se correlacionan con la sobrevida de los pacientes gran quemados ${ }^{1,4,2}$ :

- Edad.

- Sexo.

- Superficie total quemada.

Tabla 3. Comparación de 3 clasificaciones de profundidad de quemaduras. Tomada del Ministerio de Salud. Guía Clínica Gran Quemado. Minsal, 2007

\begin{tabular}{|c|c|c|c|c|}
\hline $\begin{array}{l}\text { Clasificación } \\
\text { de Benaim }\end{array}$ & $\begin{array}{l}\text { Clasificación de } \\
\text { Converswe-Smith }\end{array}$ & $\begin{array}{c}\text { Denominación } \\
\text { AB-A }\end{array}$ & Nivel histológico & Pronóstico \\
\hline $\begin{array}{l}\text { Tipo A } \\
\text { (Superficial) }\end{array}$ & Primer grado & Epidérmica & Epidermis & $\begin{array}{l}\text { No necesita injerto. Debería curar } \\
\text { espontáneamente en } 7 \text { días sin } \\
\text { secuelas }\end{array}$ \\
\hline $\begin{array}{l}\text { Tipo AB-A } \\
\text { (Intermedia) }\end{array}$ & $\begin{array}{l}\text { Segundo grado } \\
\text { superficial }\end{array}$ & Dérmica superficial & Epidermis y dermis papilar & $\begin{array}{l}\text { Debería epidermizar espontánea- } \\
\text { mente en } 15 \text { días con secuelas } \\
\text { estéticas. Si se complica puede } \\
\text { profundizarse }\end{array}$ \\
\hline $\begin{array}{l}\text { Tipo AB-B } \\
\text { (Intermedia) }\end{array}$ & $\begin{array}{l}\text { Segundo grado } \\
\text { profundo }\end{array}$ & Dérmica profunda & $\begin{array}{l}\text { Epidermis, dermis papilar y } \\
\text { reticular sin afectar fanéreos } \\
\text { profundos }\end{array}$ & $\begin{array}{l}\text { Habitualmente termina en injerto } \\
\text { con secuelas estética y/o funcional; } \\
\text { puede requerir escarectomía } \\
\text { tangencial }\end{array}$ \\
\hline $\begin{array}{l}\text { Tipo B } \\
\text { (Total) }\end{array}$ & Tercer grado & Espesor total & $\begin{array}{l}\text { Epidermis, dermis e hipo- } \\
\text { dermis (tejido celular sub- } \\
\text { cutáneo), pudiendo llegar } \\
\text { inclusive hasta el plano óseo }\end{array}$ & $\begin{array}{l}\text { Requiere escarectomía } \\
\text { precoz, e injerto o colgajos }\end{array}$ \\
\hline
\end{tabular}


Tabla 4. Clasificación de Benaim. Tomada del Ministerio de Salud. Guía Clínica Gran Quemado. Minsal, 2007

\begin{tabular}{|llcl|}
\hline \multicolumn{1}{|c}{ Características } & \multicolumn{1}{c}{$\begin{array}{c}\text { Tipo A (Superficial) } \\
\text { Figura 3 }\end{array}$} & $\begin{array}{c}\text { Tipo A-B (Intermedia) } \\
\text { Figura 4 }\end{array}$ & \multicolumn{1}{c|}{$\begin{array}{c}\text { Tipo B (Total) } \\
\text { Figura 5 }\end{array}$} \\
Aspecto clínico & $\begin{array}{l}\text { Flictenas color rojo; turgor } \\
\text { normal }\end{array}$ & $\leftrightarrow$ & $\begin{array}{l}\text { Sin flictenas; color blanco } \\
\text { grisáceo; sin turgor }\end{array}$ \\
Dolor & Intenso & $\leftrightarrow$ & Indoloro \\
Evolución & Regeneración & $\leftrightarrow$ & Escara \\
Curación por & Epidermización espontánea & $\leftrightarrow$ & Cicatrización o injerto \\
Resultado estético & Excelente & $\leftrightarrow$ & Deficiente \\
\hline
\end{tabular}

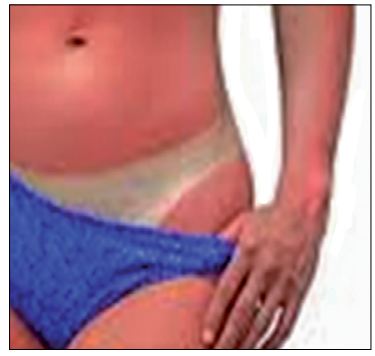

Figura 3. Quemadura Tipo A.

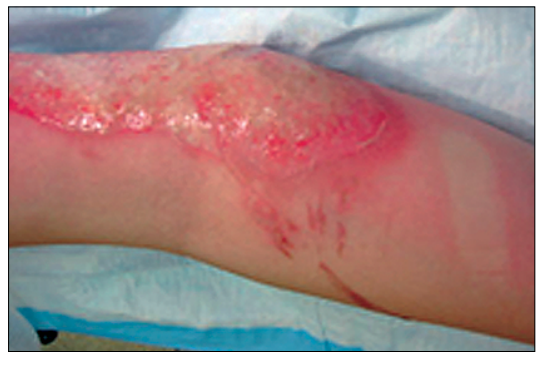

Figura 4. Quemadura Tipo AB.

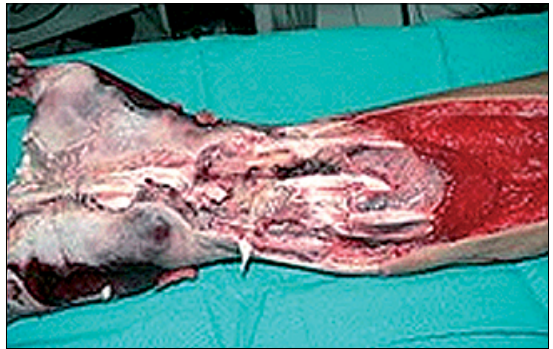

Figura 5. Quemadura Tipo B.

Tabla 5. Clasificaciones de Gravedad recomendadas según edad del paciente. Tomada del Ministerio de Salud. Guía Clínica Gran Quemado. Minsal, 2007

\begin{tabular}{|c|c|c|}
\hline Edad & Clasificación recomendada & Descripción \\
\hline Adultos & Garcés & $\begin{array}{l}\text { Edad + \% Quemadura Tipo A x } 1+\% \text { Quemadura Tipo AB x } \\
2+\% \text { Quemadura Tipo B x } 3\end{array}$ \\
\hline 2 a 20 años & Garcés modificada por Artigas & $\begin{array}{l}40 \text { - Edad + \% Quemadura Tipo A x } 1+\% \text { Quemadura Tipo } \\
\text { AB x } 2+\% \text { Quemadura Tipo B x } 3\end{array}$ \\
\hline$<2$ años & $\begin{array}{l}\text { Garcés modificada por Artigas y consenso } \\
\text { Minsal de } 1999\end{array}$ & $\begin{array}{l}40 \text { - Edad + \% Quemadura Tipo A x } 2+\% \text { Quemadura Tipo } \\
\text { AB x } 2+\% \text { Quemadura Tipo B x } 3+\text { Constante } 20\end{array}$ \\
\hline
\end{tabular}

- Profundidad de la quemadura.

- Presencia de injuria inhalatoria.

En el Servicio de Quemados del HUAP, los pacientes son calificados en términos de su gravedad, como aproximación pronóstica; probablemente esta clasificación tiene dudoso valor individual. El índice de gravedad a aplicar depende de la edad ${ }^{1}$ (Tabla 5).

De acuerdo al cálculo determinado, luego de aplicar los índices descritos, las quemaduras se clasifican según su puntaje en los pronósticos descritos en la Tabla 6.
Tabla 6. Pronóstico de quemaduras según Índices obtenidos al aplicar Clasificaciones de Gravedad. Tomada del Ministerio de Salud. Guía Clínica Gran Quemado. Minsal, 2007

\begin{tabular}{|ll|}
\hline Índice & Pronóstico \\
$21-40$ & Leve: Sin riesgo vital \\
$41-70$ & Moderado: Sin riesgo vital, salvo complicaciones \\
$71-100$ & $\begin{array}{l}\text { Grave: Probabilidad de muerte inferior a sobrevi- } \\
\text { da. Mortalidad }<30 \%\end{array}$ \\
$101-150$ & Crítico: Mortalidad $30-50 \%$ \\
$>150$ & Sobrevida excepcional: Mortalidad $>50 \%$ \\
\hline
\end{tabular}


Tabla 7. Índice de severidad de las quemaduras y pronóstico (Tobiasen et al. ${ }^{4}$ )

\begin{tabular}{|c|c|c|}
\hline Parámetro & Valor & Puntaje \\
\hline Sexo (1) & $\begin{array}{l}\text { Femenino } \\
\text { Masculino }\end{array}$ & $\begin{array}{l}1 \\
0\end{array}$ \\
\hline Edad (2) & $\begin{array}{c}<20 \\
21 \text { a } 40 \\
41 \text { a } 60 \\
61 \text { a } 80 \\
>80\end{array}$ & $\begin{array}{l}1 \\
2 \\
3 \\
4 \\
5\end{array}$ \\
\hline $\begin{array}{l}\text { Injuria inhalatoria } \\
\text { (3) }\end{array}$ & $\begin{array}{l}\text { Sí } \\
\text { No }\end{array}$ & $\begin{array}{l}1 \\
0\end{array}$ \\
\hline $\begin{array}{l}\text { Quemadura de } \\
\text { espesor total (4) }\end{array}$ & $\begin{array}{l}\text { Sí } \\
\text { No }\end{array}$ & $\begin{array}{l}1 \\
0\end{array}$ \\
\hline $\begin{array}{l}\% \text { superficie } \\
\text { total corporal } \\
\text { comprometida (5) }\end{array}$ & $\begin{array}{c}<10 \\
11 \text { a } 20 \\
21 \text { a } 30 \\
31 \text { a } 40 \\
41 \text { a } 50 \\
51 \text { a } 60 \\
61 \text { a } 70 \\
71 \text { a } 80 \\
81 \text { a } 90 \\
>90\end{array}$ & $\begin{array}{r}1 \\
2 \\
3 \\
4 \\
5 \\
6 \\
7 \\
8 \\
9 \\
10\end{array}$ \\
\hline $\begin{array}{l}\text { Índice total de } \\
\text { quemaduras }\end{array}$ & $1+2+3+4+5=$ & \\
\hline $\begin{array}{l}\text { Índice total de } \\
\text { quemaduras }\end{array}$ & $\begin{array}{l}\text { Amenaza a la } \\
\text { vida }\end{array}$ & $\begin{array}{c}\text { Probabilidad de } \\
\text { Sobrevida \% }\end{array}$ \\
\hline $\begin{array}{l}2 \text { a } 3 \\
4 \text { a } 5 \\
6 \text { a } 7 \\
8 \text { a } 9 \\
10 \text { a } 11 \\
12 \text { a } 13\end{array}$ & $\begin{array}{c}\text { Muy baja } \\
\text { Moderada } \\
\text { Moderada a } \\
\text { severa } \\
\text { Seria } \\
\text { Severa } \\
\text { Máxima }\end{array}$ & $\begin{array}{c}99 \\
98 \\
80 \text { a } 90 \\
50 \text { a } 70 \\
20 \text { a } 40 \\
<10\end{array}$ \\
\hline
\end{tabular}

Los Índices de Gravedad antes expuestos, no consideran factores como el sexo y la presencia de injuria inhalatoria. El ABSI (Abbreviated Burn Severity Index), desarrollado por Tobiasen, los considera ${ }^{4}$ (Tabla 7).

La mortalidad de los pacientes quemados también está influenciada por la existencia de comorbilidad significativa y por demoras en la reanimación ${ }^{6}$.

\section{Fecha del accidente}

El tiempo transcurrido entre ocurrida la quemadura y el tratamiento quirúrgico para resecar las escaras es uno de los factores que influye en el sangrado perioperatorio ${ }^{6}$.

\section{Agente que provocó la quemadura}

Las quemaduras son causadas por:

- Agentes físicos (85\%): llamas, líquidos u objetos calientes, radiación, corriente eléctrica, frío.

- Agentes químicos (12\%): cáusticos.

- Agentes biológicos (3\%): medusas, reptiles, insectos, batracios, vegetales.

\section{Presencia de shock inicial}

Es importante determinar si se ha superado la etapa de shock inicial del gran quemado, como requisito para someterlo a una intervención quirúrgica. Al menos debemos estar a tiempo con la fórmula escogida para reanimar y considerar diversos criterios de reanimación apropiada. Demoras en la reanimación con fluidos aumentan la incidencia de falla renal y la mortalidad ${ }^{4}$. Una de las fórmulas más populares, la fórmula de Parkland ya citada anteriormente, es la utilizada en el Servicio de Quemados del HUAP. Debe administrarse la mitad del volumen calculado dentro de las primeras $8 \mathrm{~h}$ después del accidente y la otra mitad dentro de las $16 \mathrm{~h}$ siguientes, a flujo continuo.

Criterios de reanimación apropiada ${ }^{6}$ :

- Normalización de la presión arterial.

- Débito Urinario $\left(1-2 \mathrm{ml} \cdot \mathrm{kg}^{-1} \cdot \mathrm{h}^{-1}\right)$.

- Lactato $\left(<2 \mathrm{mmol} \cdot \mathrm{L}^{-1}\right)$.

- Déficit de base $(<-5)$.

- pH gástrico intramucosa $(<7,32)$.

- Presión venosa central (tendencia).

- Índice cardiaco IC $\left(4,5 \mathrm{~L} \cdot \mathrm{min} \cdot \mathrm{m}^{-2}\right)$.

- Índice de entrega de oxígeno $\mathrm{DO}_{2} \mathrm{I}(600 \mathrm{ml}$. $\min ^{-1} \cdot \mathrm{m}^{-2}$ ).

\section{Presencia de injuria inhalatoria}

Hay consenso en que la injuria inhalatoria es un factor que agrava el pronóstico del gran quemado $^{1,4,8}$. A pesar de ello, el índice de gravedad que se aplica en el Servicio de Quemados del HUAP, no da cuenta de este factor. Se sospecha quemadura respiratoria o injuria inhalatoria cuando hay presencia de $\mathrm{de}^{1,2,4,6,8,17}$ :

- Historia clínica sugerente:

- Accidente en sitio cerrado.

- Existencia de compromiso de conciencia.

- Materiales involucrados en el incendio (plásticos, químicos).

- En el examen físico:

- Quemaduras faciales.

- Vibrisas quemadas. 
- Lesiones de mucosa orofaríngea.

- Esputo carbonáceo.

- Estridor fundamentalmente inspiratorio.

- Ronquera.

- Polipnea.

- Disnea.

- Desorientación.

- Hipoxemia, crepitaciones y sibilancias.

- Superficie total quemada superior al $30 \%$.

- Gasometría: puede inicialmente ser normal o mostrar hipoxemia y/o retención de $\mathrm{CO}_{2}$.

- Niveles de carboxihemoglobina superiores a $10 \%$ y niveles tóxicos de cianuro; ambos son componentes tóxicos del humo.

\section{Consideración del plan quirúrgico}

La evaluación preanestésica no puede ser completa si no considera el plan quirúrgico. Los diversos tipos de cirugías a las que se somete el paciente quemado, su extensión y sus diferentes localizaciones en la anatomía del paciente, tienen distintos requerimientos respecto de reserva de hemoderivados, prevención de hipotermia, dificultades en monitorización, etc. En general, las escarectomías son cirugías que determinan importantes sangrados intraoperatorios y que requieren de un mayor cuidado de prevención de hipotermia. Las amputaciones de extremidades no siempre presentan grandes sangrados, sin embargo, pueden determinar un secuestro significativo de la volemia del paciente, por lo que también pueden requerir de reserva de hemoderivados.

También es importante considerar el carácter de urgencia de la cirugía, de modo de realizar una evaluación preanestésica acotada a esa condición.

Las cirugías más frecuentes a las que se somete el paciente gran quemado, que tienen como objetivo el tratamiento de sus quemaduras son:

\section{Aseo quirúrgico inicial}

Permite:

- Establecer diagnóstico de extensión, profundidad, compresión y restricción de las quemaduras.

- Establecer un pronóstico inicial.

- Realizar las intervenciones mínimas para asegurar la estabilidad y supervivencia del paciente:

- Escarotomía: es un procedimiento de urgencia que está indicado en las quemaduras profundas, circulares que comprometen la circulación distal (llene arterial y retorno venoso).

- Fasciotomía: es un procedimiento de urgencia que está indicado en quemaduras eléctricas de alta tensión, que comprometen la circulación distal y generan síndrome de compartimento del segmento o de grupos musculares específicos. También está indicada en síndromes compartimentales asociados a sobrecarga en la reanimación, cuando otras medidas no muestran resultados.

- Descompresión de tronco: es un procedimiento de urgencia que está indicado en las quemaduras profundas de tórax que comprometan $2 / 3$ de su contorno y generen restricción respiratoria, y en las quemaduras de pared abdominal que produzcan restricción respiratoria y/o síndrome de compartimiento abdominal.

Los tres procedimientos anteriormente descritos son medidas de urgencia que deben realizarse en quirófano, generalmente por cirujanos generales, bajo anestesia, una vez realizado el aseo quirúrgico inicial y establecido el diagnóstico de extensión y profundidad. No corresponde realizar, en esta etapa, escarectomías, amputaciones ni otras cirugías de la especialidad, ni de mayor envergadura, salvo que fueren necesarias para la sobrevida del paciente ${ }^{1}$.

\section{Escarectomías tangenciales con coberturas transitorias o permanentes}

\section{Escarectomías a fascia con coberturas transitorias o permanentes}

\section{Aseos quirúrgicos posteriores}

\section{Amputación de ortejos o dedos}

\section{Amputación de extremidades a distintos niveles}

\section{Recomendaciones generales:}

Todos los aseos quirúrgicos y curaciones deben ser realizados en pabellón, bajo anestesia y respetando condiciones de asepsia y antisepsia. Esto, que parece de sentido común, no siempre era una práctica habitual. Hace no muchos años atrás se realizaban curaciones en sala, bajo dudosas condiciones de esterilidad y sometiendo al paciente a un sufrimiento que constituía un aten- 
tado a su dignidad.

Prevenir hipotermia: se debe trabajar en un pabellón climatizado a mayores temperaturas que una cirugía general o de otras especialidades, acotando los tiempos quirúrgicos y poniendo en práctica las medidas probadamente eficientes para el control de hipotermia.

Aprovechar la anestesia general, y por lo tanto, la presencia del anestesiólogo y enfermera de pabellón, para realizar procedimientos invasivos (accesos vasculares, sonda nasoyeyunal, ecocardiografía transesofágica, sonda Foley).

No está indicada la profilaxis antibiótica de rutina en estos pacientes, pero debe respetarse el horario de los tratamientos antibióticos indicados, en la medida que coincidan con el tiempo de la intervención.

En términos generales, una cirugía prolongada en tiempo y extensa en superficie, es mejor tolerada si el paciente ha recibido una reanimación apropiada $^{6}$.

\section{Consideración de la comorbilidad del paciente}

Al igual que los pacientes que se someten a otro tipo de intervenciones quirúrgicas, los gran quemados son portadores de todo tipo de enfermedades. Debe seguirse con ellos la misma conducta que con todos los pacientes quirúrgicos. Determinar si sus patologías se encuentran bajo control y tratamiento apropiados, o si requieren de algún estudio, control y/o tratamiento adicional, pudiendo requerir de la evaluación de otros especialistas. La prevalencia de patología psiquiátrica (incluido alcoholismo y drogadicción) y neurológica (especialmente epilepsia) en estos pacientes es muy frecuente. Dentro de este rubro debe también investigarse el antecedente de hospitalizaciones y cirugías previas, al mismo tiempo que incidentes anestésicos durante su curso.

\section{Evaluación por sistemas}

\section{Respiratorio}

El daño pulmonar del quemado puede ser producto de $2,4,8$ :

- Injuria inhalatoria, que puede causar daño por

3 mecanismos:

- Daño térmico directo, quemadura de mucosa respiratoria.

- Inflamación o irritación química.
- Toxicidad mediada por proceso inflamatorio sistémico.

- Aumento del agua extravascular; debe recordarse que están en un estado hipercatabólico, con hipoalbuminemia y disminución de presión oncótica plasmática, junto con aumento de permeabilidad de la microcirculación.

- Barotrauma inducido por ventilación mecánica.

- Daño pulmonar inducido por transfusiones sanguíneas: TRALI (transfusion-related acute lung injury).

- Neumonía: la injuria inhalatoria aumenta al doble el riesgo de sobreinfección.

- Complicaciones mecánicas de punciones venosas centrales.

- Neumotórax a tensión y contusión pulmonar en pacientes quemados producto de explosiones.

El daño pulmonar se manifiesta clínicamente por deterioro del intercambio gaseoso, reducción de la compliance pulmonar y torácica y aumento de la resistencia vascular pulmonar.

Si el paciente se encuentra en falla respiratoria severa, se sugiere revisar con el equipo de intensivistas si es posible optimizar la terapia respiratoria en el preoperatorio: nuevas estrategias ventilatorias, kinesioterapia respiratoria, broncodilatadores, antibióticos apropiados a cultivos y antibiogramas, fibrobroncoscopia diagnóstica y de aseo, pleurostomías, terapias depletivas de agua, en ocasiones extremas ECMO (extracorporeal membrane oxigenation), etc. Se aconseja implementar medidas para un traslado seguro a pabellón de aquellos pacientes en falla respiratoria grave o catastrófica, que tienen altos requerimientos de oxígeno y de PEEP. Deben ser trasladados con ventilador de transporte que permita aportar estas necesidades, al mismo tiempo que con una adecuada monitorización respiratoria y hemodinámica portátil.

\section{Cardiovascular}

El paciente gran quemado sufre cambios cardiovasculares significativos ${ }^{6,8}$. Inicialmente, aproximadamente las primeras $24 \mathrm{~h}$, se encuentra hipovolémico como consecuencia de una pérdida masiva de plasma hacia los tejidos quemados y un aumento de la permeabilidad vascular sistémica $^{2,6,8,16,17}$. De allí la importancia de la reanima- 
ción inicial con fluidos cristaloides isotónicos. La ya mencionada fórmula de Parkland es una de las más utilizadas. Posteriormente, durante las segundas $24 \mathrm{~h}$, se recomienda mantener un flujo continuo de cristaloides y agregar bolos de coloides en caso necesario. Aunque aún no se ha determinado el mejor objetivo para la reanimación del gran quemado, la recomendación actual es mantener un flujo urinario de 30 a $50 \mathrm{ml} \cdot \mathrm{h}^{-1}$ ó 0,5 $\mathrm{ml} \cdot \mathrm{kg}^{-1} \cdot \mathrm{h}^{-1}$. Este estado de shock se caracteriza por un débito cardiaco bajo, una resistencia vascular sistémica elevada e hipoperfusión tisular. La disminución en el gasto cardiaco se atribuye a hipovolemia, factores humorales sistémicos, disminución de respuesta a catecolaminas y disminución de la contractilidad por flujo coronario disminuido $^{2,6,8,16}$.

Es frecuente que los pacientes quemados reciban más volumen que el calculado a través de la fórmula de Parkland, durante la reanimación inicial (fluid creep). Esta sobrecarga determina complicaciones como edema pulmonar, síndrome compartimental abdominal y síndrome compartimental de extremidades ${ }^{6,8,16,17}$.

Después de las primeras $48 \mathrm{~h}$ de ocurrida la quemadura, comienza la fase de recuperación. Se trata de un estado hiperdinámico, caracterizado por un aumento del gasto cardiaco, disminución de la resistencia vascular sistémica y taquicardia. Durante esta fase se llevarán a cabo la mayor parte de las intervenciones quirúrgicas a las que se somete el paciente gran quemado, excepto el aseo quirúrgico inicial.

El paciente que ha sido víctima de quemaduras eléctricas, tiene riesgo de presentar arritmias y daño miocárdico directo. Debe ser controlado en forma estricta evaluando cambios en ECG y arritmias. Los más frecuentes son alteraciones inespecíficas del segmento ST y onda $\mathrm{T}$ y fibrilación auricular ${ }^{17,19}$.

Resulta crucial entonces, para el anestesiólogo, evaluar si la reanimación inicial del paciente gran quemado ha sido apropiada y oportuna, tanto como el estado hemodinámico del paciente, cuantificando volumen de fluidos recibidos, diuresis, necesidad y dosificación de drogas vasoactivas, necesidad de monitorización hemodinámica invasiva.

\section{Renal}

La falla renal aguda del paciente gran que- mado ocurre más frecuentemente mientras más extensas sean sus quemaduras y en pacientes portadores de injuria inhalatoria. Puede presentarse precozmente, dentro de los primeros 5 días de ocurrido el accidente o tardíamente, desde el sexto día en adelante. La falla renal aguda precoz se atribuye a hipovolemia, a mediadores inflamatorios, a mioglobinuria y a disfunción cardiaca, mientras que la tardía a sepsis y a drogas nefrotóxicas. Puede prevenirse con una adecuada y oportuna reanimación con fluidos, escarectomía precoz y profilaxis de infecciones. La evaluación preanestésica de la función renal es fundamental para plantear un plan anestésico apropiado. Se evaluarán: función renal propiamente tal, débito urinario, volemia, electrolitos plasmáticos y urinarios, terapia diurética, dependencia de diálisis y su coordinación con el momento de la cirugía $^{2,6,17,20}$.

\section{Hematológico}

El sistema hematopoyético también reacciona frente a la intensa respuesta hipermetabólica e hiperdinámica después de una quemadura. Esta reacción determina inmunomodulación y anemia. En la fase aguda, el paciente quemado se encuentra hemoconcentrado por hipovolemia $\mathrm{y}$ fuga capilar, lo que determina hematocrito $\mathrm{y}$ viscosidad elevados. Luego, durante el período de reanimación se advierte anemia por dilución. Durante el período postagudo, la anemia es consecuencia de pérdidas perioperatorias y de la patología crítica $^{17,18}$. Para que el injerto prenda apropiadamente, la herida debe prepararse de forma que quede vascularizada óptimamente. La única forma de percibir que el tejido sobre el que se deposita el injerto está vital es que sangre. Las pérdidas de sangre asociadas a esta cirugía pueden ser estimadas de varias formas, pero lo más simple y realista es la estimación que hace un equipo de cirujanos y anestesiólogos experi$\operatorname{mentados}^{18}$.

Los pacientes gran quemados también experimentan una anemia prolongada propia de las enfermedades críticas. Se estima que sobre el $50 \%$ de las transfusiones de glóbulos rojos que reciben durante su hospitalización se atribuyen a esta causa. Este tipo de anemia es multifactorial. Factores iatrogénicos como flebotomías, tomas de muestras sanguíneas para exámenes innecesarios, cambios de apósitos o eritropoyesis difi- 
cultada por déficit nutricional y disfunción de la médula ósea ${ }^{18}$.

La evaluación preanestésica debe pesquisar la anemia, corregirla y reservar una cantidad razonable de unidades de glóbulos rojos según umbrales de transfusión y cálculo de pérdidas que se señalan más adelante. La implementación de estrategias de transfusión restrictivas disminuyen las infecciones, los costos y mejoran la sobrevida ${ }^{2,18}$.

Durante los primeros 2 días después de la quemadura, los pacientes se presentan con leucocitosis a expensas de los granulocitos. Esta se debe a la pérdida aguda de plasma, recuperación de neutrófilos maduros desde vasos periféricos y a una rápida liberación de las reservas de la médula ósea. Luego de este período, frecuentemente se desarrolla leucopenia asociada a fallas en la hematopoyesis, uso de sulfadiazina de plata local y pacientes portadores de infecciones, especialmente sepsis a Gram negativos ${ }^{18}$.

Las alteraciones de la coagulación pueden ocurrir tempranamente después de la quemadura. Los quemados presentan trombocitopenia, coagulación intravascular diseminada y otras coagulopatías, que aumentan los requerimientos de hemocomponentes perioperatorios.

La trombocitopenia que requiere de transfusión plaquetaria no es común en el gran quemado. Si es temprana puede deberse a secuestro (consumo durante la formación de microtrombos sistémicos) y a dilución por reanimación. Tardíamente es consecuencia de la sepsis ${ }^{17,18}$. Luego de 2 a 3 semanas de la quemadura, se identifica un período de aumento de la producción plaquetaria, por lo que las plaquetas recuperan sus niveles, incluso llegando a trombocitosis. Debido a que las plaquetas se almacenan a temperatura ambiente, su contaminación bacteriana es superior a la de otros hemocomponentes, por lo que su uso debe ser limitado, dadas las complicaciones infecciosas.

Los cambios en la coagulación observados en gran quemados son transitorios y se previenen con una apropiada reanimación y resolución de la fase aguda de la respuesta a la injuria. Durante el período inicial de reanimación los factores y proteínas de la coagulación disminuyen por un efecto dilucional y por la pérdida de proteínas plasmáticas al intersticio. Posteriormente pueden contribuir a su pérdida un sangrado masivo perioperatorio, o una función hepática comprometida. Las coagulopatías clínicamente significativas no son frecuentes. Durante el período de recuperación de la quemadura, la coagulopatía está menos relacionada con la quemadura misma y se explica por aspectos más relacionados con los cuidados críticos, como es la sepsis.

La evaluación de la coagulación que es posible realizar en el HUAP, es bastante rudimentaria. Se dispone de tiempo de protrombina, INR, TTPK, recuento plaquetario y fibrinógeno plasmático. Para una evaluación más precisa, desde el inicio de la formación del coágulo hasta su lisis, se recomienda contar con métodos viscoelásticos.

\section{Endocrino, metabólico y nutricional}

Las quemaduras superiores a un $40 \%$ de la superficie corporal total, se acompañan inmediatamente después del accidente, de un período de estrés, inflamación e hipercatabolismo. Esta respuesta no es sólo típica del gran quemado; la presentan también los pacientes que sufren un traumatismo considerable, una gran cirugía o cualquier enfermedad crítica. Sin embargo, la severidad, duración y magnitud con que ocurre en el gran quemado, la hacen particular ${ }^{13}$.

La tasa metabólica del gran quemado aumenta, con un máximo entre los 7 a 12 días después del accidente, momento en que puede doblar la tasa habitual. Este estado hipermetabólico determina un importante catabolismo proteico con pérdida de masa muscular, que deteriora la inmunidad y demora la cicatrización y curación de heridas. Estrategias para mitigar esta gran demanda metabólica son: un tratamiento precoz de las quemaduras, mantener un ambiente cálido $\left(28-33^{\circ} \mathrm{C}\right)$, agentes farmacológicos como insulina y $\beta$ bloqueadores y soporte nutricional ${ }^{2,4-6}$. $\mathrm{La}$ alimentación enteral es la forma óptima de alimentar al gran quemado. Ésta, además de aportar nutrientes, ayuda a la mantención de la barrera intestinal, previniendo translocación bacteriana y por hongos. Al mismo tiempo facilita el control de las agresiones sépticas, asociándose a sobrevidas muy superiores a la alimentación parente$\mathrm{ral}^{4-6,15}$.

El paciente gran quemado debe someterse a varias intervenciones quirúrgicas. El hecho de tener que dejarlo en ayuno por extensos períodos pre y postoperatorios, interfiere significa- 
tivamente con un apropiado aporte de sus necesidades nutricionales y determina un déficit calórico. Esta es la razón por la que se nos solicita limitar o suspender los períodos de ayuno perioperatorio, sin exponer a mayor riesgo de aspiración pulmonar de contenido gástrico a estos pacientes $^{6,9-12,14,15,17}$. Una temperatura ambiental apropiada, también ayuda a modular el hipercatabolismo. Como resultado de este, los pacientes presentan un aumento en el consumo de oxígeno y una producción de $\mathrm{CO}_{2}$ también elevada. Ambos demandan un mayor esfuerzo respiratorio.

Resistencia a insulina, hiperinsulinemia e hiperglicemia son las alteraciones metabólicas claves del gran quemado, que se benefician de un estricto control glicémico, el que, aunque no ha sido documentado para el intraoperatorio, se asume igualmente provechoso.

\section{Problemas específicos a considerar}

\section{Lesiones asociadas a la quemadura}

De la misma forma que cualquier víctima de un traumatismo severo, el paciente quemado debe recibir a su ingreso a un servicio de urgencia los siguientes cuidados: asegurar permeabilidad de vía aérea, oxigenación, ventilación y mantención de circulación (prioridades ATLS) ${ }^{4,8}$. Debe evaluarse la presencia de lesiones asociadas a la quemadura, siendo las fracturas las más frecuentes. En víctimas de explosiones, se puede encontrar neumotórax a tensión y contusión pulmonar. De acuerdo a las características del accidente, también se evaluará la columna cervical. En caso de compromiso del sensorio, debe evaluarse por neurocirugía. Secundariamente se abordará el problema específico de la quemadura. La evaluación preanestésica deberá considerar estos hallazgos asociados y verificar que su diagnóstico y tratamiento estén ya implementados.

\section{Ayuno preoperatorio}

El paciente que se alimenta con alimentación enteral distal al píloro, duodenal o en yeyuno, puede ser intervenido quirúrgicamente en forma segura, sin detener su alimentación ${ }^{6,10-12,14,17}$. Esta práctica debe ser acompañada de una monitorización diligente de la posición de la sonda de alimentación, cercana al día de la operación y de una evaluación clínica permanente de la tolerancia del paciente a su alimentación. La American
Burn Association, también recomienda este tipo de alimentación, pues no requiere ser interrumpida en relación a procedimientos o intervenciones quirúrgicas, lo que constituye un avance significativo en el aporte de sus requerimientos nutricionales. Hay centros en que incluso continúan la alimentación enteral intraoperatoria, a través de sonda naso-gástrica en el paciente intubado, sin haberse observado complicaciones como aspiración de contenido gástrico ${ }^{9}$. Sin embargo, es más utilizada y más prudente la primera recomendación.

\section{Manejo de la vía aérea}

En la mayoría de los casos el paciente gran quemado ingresará a pabellón intubado. En su período más crítico se encuentra en intensivo, en ventilación mecánica. Pueden ser excepciones la primera cirugía, de aseo quirúrgico inicial, o las últimas cirugías de cobertura de zonas residuales, en que el paciente se encuentra prácticamente recuperado.

Pese a esto, no es infrecuente que al anestesiólogo se le solicite ayuda con el manejo inicial de la vía aérea, que debamos cambiar un tubo endotraqueal durante el período perioperatorio o que durante el traslado del paciente a pabellón se extube y requiera reintubación.

Las quemaduras de cara, cuello y tórax superior pueden distorsionar la anatomía y, durante el período agudo postinjuria, causar obstrucción de la vía aérea, además de dificultar la ventilación con mascarilla facial, la laringoscopia e intubación debido a $\mathrm{a}^{2,4,6,8}$ :

- Edema de labios, lengua, orofaringe y laringe que obstruyen la vía aérea.

- Quemadura y edema de partes blandas del cuello y submandibulares que comprometen la compliance del espacio submandibular y la movilidad del cuello.

- Limitación de la apertura bucal.

La evaluación preanestésica debe ir dirigida a buscar predictores de vía aérea difícil. De estar presentes, debemos diseñar planes alternativos para el manejo de la vía aérea que deben considerar los diversos instrumentos disponibles para ello. El manejo debe incluir un período considerable de preoxigenación. Estos pacientes tienen una reserva respiratoria muy limitada, con disminución de la CRF que, durante un período 
de apnea, determinará una precoz y brusca disminución de la saturación de la hemoglobina, comprometiéndose su oxigenación. En ocasiones, con ayuda de un cirujano es posible ampliar la apertura bucal a través de una comisurotomía uni o bilateral, o mejorar el rango de movilidad del cuello realizando una o más incisiones en la escara o en la cicatriz del cuello causante de su limitada movilidad.

Debe tenerse presente siempre el riesgo del uso de relajantes musculares para facilitar la intubación en pacientes que no se tiene seguridad de poder ventilar o intubar. Esta situación es extremadamente poco probable en la actualidad, al disponer de una serie de dispositivos alternativos para el manejo de la vía aérea difícil y además no es válida sólo para pacientes quemados, pues puede ocurrir en diversas situaciones clínicas. Se recomiendan técnicas con el paciente despierto, en ventilación espontánea ${ }^{2,4,6}$. Cricotiroidotomía y traqueostomía de emergencia deben estar disponibles en caso de fallar las técnicas no invasivas.

Se contraindican dispositivos supraglóticos (mascarilla laríngea, tubo laríngeo) en caso de edema masivo de vía aérea superior ${ }^{4}$. Durante el período reciente después de la quemadura, el edema masivo de la vía aérea sumado a una apertura bucal limitada, impiden la introducción de estos dispositivos. Insistir en esta maniobra puede causar aún mayor daño en una mucosa y tejidos blandos de vía aérea que se encuentran extremadamente friables.

Especial consideración merece el paciente que ha permanecido en posición prona en forma prolongada y que requiere de intubación o reintubación. El edema de declive es considerable y requerirá de mucha habilidad, práctica y experiencia para realizar el procedimiento exitosamente.

El paciente portador de sepsis, especialmente en su período de soporte hemodinámico y respiratorio más intenso, puede presentar vaciamiento gástrico lento, determinando residuo gástrico y riesgo de aspiración.

Durante el período de recuperación y lesiones residuales, la contractura y retracción de cara, cuello y tórax constituye el cambio más frecuente de la vía aérea y determinará limitaciones tanto en la flexo-extensión del cuello como en la apertura bucal ${ }^{8,17}$. Si la quemadura no compromete cara y cuello, puede realizarse un manejo de la vía aérea habitual ${ }^{6}$.

La fijación del tubo traqueal, en presencia de quemadura facial, requiere de una atención especial. Fijamos este dispositivo con una sutura firme a algún incisivo ${ }^{4,6}$. En caso de tratarse de pacientes desdentados, se fija con un punto a la encía.

\section{Ventilación mecánica intraoperatoria}

Se recomienda simular el modo y los parámetros de ventilación mecánica utilizados en intensivo, durante la intervención quirúrgica. En ocasiones en que el paciente esté recibiendo modalidades de ventilación mecánica especiales, y/o que su falla respiratoria sea de una gravedad considerable, el ventilador de la máquina de anestesia puede ser insuficiente para sus necesidades, razón por la que se recomienda usar el ventilador de intensivo dentro del pabellón.

\section{Disponibilidad de vías venosas}

Existen dificultades objetivas para asegurar accesos vasculares apropiados en el paciente gran quemado, especialmente en su período inicial. El shock inicial dificulta la punción de vías venosas periféricas. No es infrecuente encontrarse con el sitio de inserción de los accesos vasculares comprometido por la quemadura, con la distorsión anatómica que este hecho determina. En ocasiones, cuando las quemaduras son extensas, es necesario realizar la punción vascular a través de ellas ${ }^{6}$. La necesidad de cambiar frecuentemente la ubicación de catéteres, para minimizar el riesgo de infección, agrava el problema. Es útil el uso de ultrasonido para guiar la inserción de catéteres. Los catéteres venosos subclavios tienen menor riesgo de infección que los yugulares internos y que los femorales, pero mayor frecuencia de complicaciones mecánicas. El catéter venoso femoral no tiene complicaciones mecánicas pero tiene mayor riesgo de infección y de trombosis que el subclavio y el yugular interno. Este último ofrece dificultades de inserción cuando hay quemaduras de cara y cuello, especialmente en presencia de traqueostomía, se infecta más que el subclavio y además es incómodo, lo que puede no ser significativo si el paciente se encuentra sedado. La evaluación preanestésica debe evaluar la presencia, permeabilidad y apropiada fijación de los accesos vasculares. La anestesia es un buen momento para 
realizar cambios de accesos vasculares porque el paciente se encuentra dormido y porque se aprovechan las habilidades y destrezas del operador, que van en beneficio de menor morbilidad asociada a procedimientos.

Algunas recomendaciones al respecto ${ }^{17}$ :

- Instalar accesos vasculares sobre piel sana si es posible.

- Accesos de gran calibre son necesarios en el gran quemado que requiere reposición de volúmenes considerables durante reanimación o concomitantes a sangrados masivos perioperatorios.

- El acceso intraóseo es una alternativa en pacientes con dificultades en accesos venosos.

- Los accesos arteriales invasivos son necesarios para obtener información de la curva arterial y para obtener muestras sanguíneas.

- Los accesos venosos centrales son necesarios para el aporte de drogas vasoactivas y para evaluar tendencia de PVC.

- Aplicar la política de rotación de vías venosas centrales.

- Usar calibres pequeños disminuye el riesgo de trombosis.

\section{Dificultades en la monitorización}

Las principales dificultades con la monitorización intraoperatoria del paciente quemado, están relacionadas con el hecho de que tanto los aseos quirúrgicos como las escarectomías a los que se somete pueden incluir, durante la misma cirugía, extensas y diversas zonas anatómicas, de la parte anterior y posterior de su cuerpo. Esto tiene como consecuencia que varias o todas sus extremidades, tronco y cabeza, pueden ser parte del campo operatorio durante una cirugía. En tales casos, hay que ir cambiando de lugar los electrodos de electrocardiografía y del estimulador de nervio periférico, el sensor del oxímetro de pulso, el manguito de PANI, según el lugar don- de se realiza la cirugía, llegando incluso a perder por breves lapsos la monitorización de algunos parámetros. Todos los electrodos adhieren con dificultad debido a lesiones de piel, por lo que en caso necesario puede usarse alternativamente corchetes quirúrgicos y clips tipo cocodrilo. La monitorización más constante resulta ser la capnografía, temperatura y diuresis. Debido a lo anterior, en la evaluación preanestésica se debe evaluar en forma dirigida estas dificultades para poder anticiparnos y disponer del material apropiado a esta monitorización. Debe disponerse de material alternativo que hará las veces de electrodos, cables de oximetría de pulso largo que alcancen a todas las extremidades, sensores de oximetría de pulso para oreja y lengua, mangueras de mangos de PANI de similares características ${ }^{6,17}$.

\section{Sangrado perioperatorio}

La estimación del sangrado asociado a la intervención quirúrgica resulta crucial para la preparación preoperatoria del paciente (Tabla 8). El paciente que se someterá a cirugía de escarectomía para resecar quemaduras $\mathrm{AB}$ profundas o $\mathrm{B}$, debe enfrentar la cirugía bajo condiciones apropiadas de hemoglobina preoperatoria y de coagulación. Existe controversia respecto del umbral considerado para la transfusión del paciente gran quemado ${ }^{6}$. En el Servicio de Quemados del HUAP, el umbral perioperatorio de hemoglobina acordado para el paciente gran quemado es de 8 a $10 \mathrm{gr}^{\circ} \mathrm{dL}^{-1}$, más alto que el que pedimos al paciente general y semejante al que solicitamos para el paciente con comorbilidad cardiorespiratoria severa, el adulto mayor o el portador de isquemia miocárdica sintomática ${ }^{7}$. En general, se sugieren umbrales de transfusión de $7 \mathrm{a} 8 \mathrm{gr} \cdot \mathrm{dL}^{-1}$, cuando el paciente no presenta sangrado activo, compromiso hemodinámico o isquemia miocárdica $^{2,18}$. Como en todos los casos, una evaluación clínica del paciente es lo que mejor determinará

Tabla 8. Cálculo de pérdidas sanguíneas estimadas en una escarectomía (Tomado de Desai et al., Ann Surg 1990)

\begin{tabular}{|ll|}
\hline Procedimiento Quirúrgico & Pérdidas sanguíneas esperadas \\
\hline < 24 h de la quemadura & $0,45 \mathrm{ml} \cdot \mathrm{cm}^{-2}$ de superficie quemada \\
\hline a 3 días de la quemadura & $0,65 \mathrm{ml} \cdot \mathrm{cm}^{-2}$ de superficie quemada \\
\hline a 16 días de la quemadura & $0,75 \mathrm{ml} \cdot \mathrm{cm}^{-2}$ de superficie quemada \\
> a 16 días de la quemadura & 0,5 a $0,75 \mathrm{ml} \cdot \mathrm{cm}^{-2}$ de superficie quemada \\
Quemaduras infectadas & 1 a $1,25 \mathrm{ml} \cdot \mathrm{cm}^{-2}$ de superficie quemada \\
\hline
\end{tabular}


su necesidad de transfusión.

Entre los factores que influyen en la cuantía del sangrado perioperatorio están ${ }^{6}$ :

- El área resecada.

- El tiempo transcurrido entre el accidente y la cirugía.

- El plan quirúrgico (escarectomía tangencial $v s$ escarectomía a fascia).

- La presencia de infección.

- El sangrado de la zona dadora de injertos (zona dadora inicial o repetida de toma de injerto).

En resumen, además de enfrentar la escarectomía con una hemoglobina basal de 8 a $10 \mathrm{gr} \cdot \mathrm{dL}^{-1}$, se debe solicitar al banco de sangre una reserva del número de unidades de glóbulos rojos necesarias para cubrir el sangrado calculado. Esta petición debe ser confirmada inmediatamente antes de pasar al paciente a pabellón. Un cálculo sencillo de solicitud preoperatoria de glóbulos rojos es de 1,78 unidades por cada metro cuadrado de quemadura que será escarectomizada ${ }^{18}$.

Respecto de las condiciones de coagulación necesarias para la escarectomía, se acepta que el paciente tenga un INR menor o igual a $1,5^{17}$ $\mathrm{y}$ un recuento plaquetario de al menos 50.000 plaquetas ${ }^{17}$. Debe considerarse que se trata de sangrado al exterior, que se puede diagnosticar con facilidad y que el sitio quirúrgico es compresible con vendajes habituales y elásticos. Debe solicitarse al banco de sangre tener preparados unidades de plasma fresco congelado, plaquetas y crioprecipitados en caso de que así se requiera luego de una evaluación preoperatoria de la coagulación. Estos hemocomponentes deben ser infundidos cercanos a la cirugía y su disponibilidad debe confirmarse antes de pasar al paciente a pabellón. En caso de compromiso significativo de la coagulación, podría ser de alguna utilidad la administración de vitamina $\mathrm{K}$ endovenosa. Un rol importante tiene el uso de complejo protrombínico, especialmente en aquellos pacientes que no toleran la sobrecarga de volumen que acompaña al uso de varias unidades de plasma fresco congelado. Durante la cirugía, debe utilizarse todas las medidas disponibles con el objetivo de disminuir el sangrado.

\section{Posición postoperatoria}

Durante la evaluación preanestésica se debe detectar la posibilidad de que el paciente salga del pabellón en posiciones diferentes al decúbito dorsal. La cobertura de escarectomías de la espalda requiere de posición prona para que los injertos prendan apropiadamente. Debemos estar enterados de este requerimiento pues tiene como requisito que el paciente salga en dicha posición desde el pabellón intubado, en ventilación mecánica y bajo sedación mediana a profunda.

\section{Hipotermia}

En el paciente gran quemado, el umbral al que se desencadena la respuesta termoreguladora está elevado. Junto con esto, la respuesta de vasoconstricción cutánea, uno de los mecanismos de mantención de la temperatura central, evitando la pérdida de calor al ambiente, se compromete al perderse extensas áreas de piel producto de la quemadura y posteriormente de la escarectomía, especialmente a fascia. Esto resulta en una pérdida de calor desde el compartimento central al ambiente. Otro mecanismo de pérdida de calor en el gran quemado es la evaporación ${ }^{6}$.

La inducción y mantención de anestesia general, interrumpen los mecanismos termoregulatorios y exponen aún más al paciente a hipotermia. Esta ocurre más frecuentemente en pacientes inapropiadamente reanimados o inestables metabólicamente y predispone a intolerancia a las drogas anestésicas y al estrés quirúrgico ${ }^{6}$.

La evaluación preanestésica del paciente gran quemado debe dirigirse a pesquisar, prevenir y tratar la hipotermia. El paciente no debe entrar hipotérmico al pabellón, debiéndose implementar todas las medidas conocidas para monitorizar y tratar la hipotermia perioperatoria, partiendo por una apropiada temperatura ambiental (28 a $\left.33^{\circ} \mathrm{C}\right)^{2,6}$.

\section{Gérmenes aislados}

El paciente quemado es propenso a infecciones por múltiples razones. La falta de piel lo predispone a infección de sus heridas; la intubación $\mathrm{y} / \mathrm{o}$ traqueostomía determina un quiebre en la barrera de la vía aérea superior; la permeabilidad intestinal a bacterias se encuentra elevada; los accesos vasculares venosos y arteriales, permiten a gérmenes acceder directamente al torrente sanguíneo y además se encuentra inmunodeprimido ${ }^{17}$. La evaluación preanestésica deberá pes- 
quisar la presencia de gérmenes aislados, que hayan motivado la decisión de mantener al paciente en aislamiento de contacto. Es necesario identificarlos y verificar la duración del tratamiento antibiótico que ha recibido el paciente. Aunque todos los pacientes gran quemados se manejan en el intraoperatorio como si estuviesen en aislamiento de contacto, por las razones aportadas, hay algunos gérmenes, que al terminar la cirugía, motivan el aseo terminal de pabellón. Este tiene una duración extensa, atentando contra el normal desarrollo de la tabla quirúrgica, razón por la que estos pacientes deben ser programados a la última hora.

\section{Conclusiones}

La evaluación preanestésica del paciente gran quemado, debe considerar la misma información, elementos y pasos que la de cualquier paciente quirúrgico. Pero debe, además, asumir las particularidades propias de este tipo de pacientes. Es fundamental evaluar la magnitud e impacto que en todos los órganos y sistemas provoca la respuesta inflamatoria, hipercatabólica e hiperdinámica que el organismo desencadena frente a la quemadura. Adicionalmente, hay que preparar lo mejor posible al paciente a exponerse al estrés de una cirugía de las más cruentas.

\section{Referencias}

1. MINISTERIO DE SALUD. Guía Clínica Gran Quemado. Minsal; 2007.

2. Harbin KR, Norris TE, Norris TE. Anesthetic management of patients with major burn injury. AANA J 2012 Dec;80(6):430439.

3. Protocolo Evaluación Pre Anestésica HUAP.

4. Marko P, Layon AJ, Caruso L, Mozingo DW, Gabrielli A. Burn injuries. Curr Opin Anaesthesiol 2003 Apr;16(2):183-191.

5. Gauglitz GG, Finnerty CC, Herndon DN et al. Modulation of the hypermetabolic response after burn injury. Total Burn Care: Expert Consult - Online and Print, $4 \mathrm{e}$ by David N. Herndon MD FACS. Capítulo 30.

6. Woodson LC, Sherwood ER, Aarsland A et al. Anesthesia for burned patients. Total Burn Care: Expert Consult - Online and Print, 4e by David N. Herndon MD FACS.Capítulo 14.

7. Protocolo para la indicación de transfusión de componentes sanguíneos, HUAP.

8. Kaiser HE, Kim CM, Sharar SR, Olivar HP. Advances in Perioperative and Critical Care of the
Burn Patient. Anesthesia Management of Major Thermal Burn Injuries in Adults. Adv Anesth 2013;31(1):137-161.

9. Friese RS, Arnoldo B, Hunt J, Purdue G, Philmon W. Pre-Operative Fasting is Unnecesary for Endo-Tracheally Intubated Burn Patients. Crit Care Med 2005;33 Supplement:A41.

10. Pearson KS, From RP, Symreng T, Kealey GP. Continuous enteral feeding and short fasting periods enhance perioperative nutrition in patients with burns. J Burn Care Rehabil 1992 JulAug;13(4):477-481.

11. Sutanto LB, Surani S, Prasetyono TO et al. Role of Enteral Nutrition in Pre-operative Patients. Crit Care Shock 2009;12:95-99.

12. Bengmark S, Andersson R, Mangiante G. Uninterrupted perioperative enteral nutrition. Clin Nutr 2001 Feb;20(1):11-19.

13. Williams FN, Branski LK, Jeschke MG et al. What, how, and how much should patients with burns be fed? Surg Clin North Am 2011 Jun;91(3):609-629.

14. Prelack K, Dylewski M, Sheridan RL. Practical guidelines for nutritional management of burn injury and recovery. Burns 2007
Feb;33(1):14-24.

15. Rodríguez NA, Jeschke MG, Williams FN et al. Nutrition in burns: galveston contributions. JPEN J Parenter Enteral Nutr 2011 Nov;35(6):704-714.

16. Latenser BA. Critical care of the burn patient: the first 48 hours. Crit Care Med 2009 Oct;37(10):2819-2826.

17. Anderson TA, Fuzaylov G. Perioperative anesthesia management of the burn patient. Surg Clin North Am 2014 Aug;94(4):851-861.

18. Posluszny JA, Gamelli RL, Shankar R. Hematologic and hematopoietic response to burn injury. Total Burn Care: Expert Consult - Online and Print, 4e by David N. Herndon MD FACS. Capítulo 23.

19. Arnoldo BD, Hunt JL, Sterling JP, Purdue GF. Electrical injuries. Total Burn Care: Expert Consult - Online and Print, $4 \mathrm{e}$ by David N. Herndon MD FACS. Capítulo 38.

20. Goverman J, Aikawa N, Fagan SP. Renal failure in association with thermal injuries. Total Burn Care: Expert Consult Online and Print, 4e by David N. Herndon MD FACS.Capítulo 32. 